\title{
LHC upgrade and LHC-ATLAS upgrade
}

\section{Yasuyuki Okumura*}

International Center for Elementary Particle Physics, The University of Tokyo

E-mail: yasuyuki.okumura@cern.ch

\begin{abstract}
One of the most important goals in the physics programs of the Energy Frontier research is to discover hints for extensions of the Standard Model of the particle physics. The High-Luminosity Large Hadron Collider (HL-LHC) will start the operation in 2026 to sustain and extend the physics sensitivity in the new physics search at the LHC. The HL-LHC will be realized by a full upgrade of the components of the LHC accelerator complex, such as the injector chain, the collimators, and the elements in the insertion regions for the experiments. Together with the upgrade of the LHC accelerator complex, the experimental detectors will be upgraded to cope with the high luminosity environment. The ATLAS experiment at the LHC will introduce new detectors, electronics, and trigger components to sustain the high performance of the reconstruction of the proton-proton collision events. There are two classes of the major challenges in the LHC-ATLAS upgrade projects; (1) development of a new pileup robust detector system which can distinguish interesting events from the other simultaneous proton-proton collisions, and (2) development of a new Trigger and Data Acquisition system to sustain the stability of the data-taking and flexibility of the online selection criteria in the trigger system during the HL-LHC operation.
\end{abstract}

The 4th KMI International Symposium (KMI2019)

18-20, February 2019

Nagoya, Japan

${ }^{*}$ Speaker. 


\section{High-Luminosity Large Hadron Collider (HL-LHC)}

Since the Higgs boson was discovered by the ATLAS [1] and CMS [2] experiments at the Large Hadron Collider [3], one of the most important goals in the physics programs of the Energy Frontier research is to discover hints for extensions of the Standard Model of the particle physics, which is motivated by the presence of the Higgs boson in the electroweak scale. The LHC has been increasing the center-of-mass energy of proton-proton collisions and the luminosity to maximize the chance to observe unknown new phenomena during the successful physics runs through the Run 1 (2010-2013) and Run $2(2015-2018)$ at $\sqrt{s}=7,8,13 \mathrm{TeV}$. As the LHC will be the energy frontier instruments for the next two decades at $\sqrt{s}=14 \mathrm{TeV}$, increasing the instantaneous luminosity of the LHC will be the key strategy to sustain and extend the physics sensitivity in the new physics search. The High-Luminosity Large Hadron Collider (HL-LHC) [4] will start the operation in 2026, aiming to achieve the total integrated luminosity of $3000 \mathrm{fb}^{-1}$ with $250 \mathrm{fb}^{-1}$ accumulated per year. During the second long shutdown of the LHC (LS2) (2019-2020), the LHC injector system will be upgraded, and after the LS3 (2024-2026) all the LHC accelerator complex will be compatible with the HL-LHC requirements. Together with the upgrade of the LHC, the experimental detectors will be upgraded to cope with the high luminosity environment.

\section{LHC upgrade}

The luminosity of the LHC can be described with the equation of $L=\gamma \frac{n_{\mathrm{b}} N^{2} f_{\mathrm{rev}}}{4 \pi \beta^{*} \varepsilon_{\mathrm{n}}} R$, where $\gamma$ is the relativistic gamma factor of the circulating protons; $n_{\mathrm{b}}$ is the number of bunches per beam; $N$ is the number of protons per bunch; $f_{\text {rev }}$ is the revolution frequency; $\beta^{*}$ is the beta function at the interaction point; $\varepsilon_{\mathrm{n}}$ is the transverse normalized emittance; $R$ is a luminosity geometrical reduction factor due to the crossing angle [4]. The high instantaneous luminosity of the HL-LHC will be realized by the increase of the beam current and squeezing the beam size at the collision points. The upgrade of the injector chain of the LHC allows the higher beam brightness, which is defined as the ratio of the beam intensity to its transverse emittance. The number of protons per bunch will be increased by a factor of two compared with the nominal LHC design, while the emittance will be reduced. Low $\beta^{*}$ will be achieved by the upgrade of the insertion region magnet systems with an increase in the quadrupole aperture and the magnetic fields. A quadrupole magnet system with $12 \mathrm{~T}$ magnetic field will be exploited with $\mathrm{Nb}_{3} \mathrm{Sn}$ superconductors. New superconducting radiofrequency crab cavities for beam rotation with ultra-precise phase control better than 0.001 degrees will be installed around the ATLAS and CMS collision points to realize effective head-on collisions with the kick voltage of 3.4 MV. It helps the HL-LHC to sustain the geometric reduction factor $R$ while the crossing angle will increase for the very small $\beta^{*}$. To control the beam quality, an upgrade of the collimation system is also required. Upgraded collimators with a new material that produces less electromagnetic interference on the beam will replace about 60 out of the 118 existing ones. Furthermore, additional collimators will be installed at the dispersion suppressor regions, which requires replacement of a part of LHC dipoles $(8.3 \mathrm{~T}, 14.2 \mathrm{~m})$ with $\mathrm{Nb}_{3} \mathrm{Sn}$ superconducting magnets (11 T, a pair of two $5.5 \mathrm{~m}$ dipoles) to keep the equal bending strength. Although the upgraded LHC accelerator complex could reach a peak luminosity of $2.0 \times 10^{35} \mathrm{~cm}^{-2} \mathrm{~s}^{-1}$, energy deposit at the magnets in the interaction regions and needs of a smooth operation of the detector system require 


\begin{tabular}{lcc}
\hline parameters & Nominal LHC & HL-LHC \\
\hline Beam energy $(\mathrm{TeV})$ & 7 & 7 \\
Particles per bunch, $N\left(10^{11}\right)$ & 1.15 & 2.2 \\
Number of colliding bunches at ATLAS and CMS, $n_{\mathrm{b}}$ & 2808 & 2736 \\
$\beta^{*}$ at ATLAS and CMS $(\mathrm{m})$ & 0.55 & 0.2 \\
Transverse emittance, $\varepsilon_{\mathrm{n}}(\mu \mathrm{m})$ & 3.75 & 2.50 \\
Geometrical reduction factor, $R$ & 0.836 & 0.715 \\
Peak luminosity, $L\left(10^{34} \mathrm{~cm}^{-2} \mathrm{~s}^{-1}\right)$ & 1 & 5.23 \\
Average number of interactions per crossing at peak luminosity, $\langle\mu\rangle$ & 27 & 140 \\
\hline
\end{tabular}

Table 1: HL-LHC machine parameters for the standard operation with $25 \mathrm{~ns}$ bunch spacing [4]. The $R$ for the HL-LHC is for a scenario with crab cavities. The $L$ and $\langle\mu\rangle$ for the HL-LHC are for the levelled luminosity with the luminosity levelling technique.

a limitation on the peak luminosity. A so-called luminosity levelling technique allows a constant instantaneous luminosity of $5 \times 10^{34} \mathrm{~cm}^{-2} \mathrm{~s}^{-1}$ instead of a quickly decreasing luminosity profile. It allows protecting machines as well as high operational efficiency of the detector systems with the constant lower peak luminosity while almost the same average luminosity per fill will be achieved. Table 1 summarizes the HL-LHC machine parameters.

\section{LHC-ATLAS upgrade}

It is also challenging to build a detector system which is robust in the HL-LHC environment while the luminosity levelling techniques will help the detector system to realize a stable operation. This section will present the upgrade project of the ATLAS detector system for the HLLHC $[6,7,8,9,10,11,12]$, which is called the "Phase-2 upgrade". In the project, the peak luminosity is considered to ultimately reach to $7.5 \times 10^{34} \mathrm{~cm}^{-2} \mathrm{~s}^{-1}$, which corresponds to 200 simultaneous collisions per bunch crossing (pileup collisions). The Phase-2 ATLAS detector system is required to be a pileup robust system without degradation of the performance with reliable datataking capability in such a dense environment. We have two classes of the major challenges in the Phase-2 upgrade; (1) development of a new pileup robust detector system which can distinguish interesting events from the other 200 pileup collisions, and (2) development of a new Trigger and Data Acquisition (TDAQ) system to sustain the stability of the data-taking and flexibility of the online selection criteria in the trigger system during the HL-LHC operation.

\subsection{Development of a new pileup robust detector system}

Excellent performance of charged particle tracking and vertex reconstruction is a key requirement for the success of the HL-LHC physics programs. A design of all-silicon central tracking system is found to be a natural solution for the new ATLAS Inner Tracker (ITk) of the Phase-2 system [6, 7]. The ITk consists of a Pixel Detector at a small radius and a large-area outer Strip Detector. The Strip Detector has four barrel layers and six end-cap petal-design disks, covering $|\eta|<2.7$ with double-sided modules for a stereo angle measurement capability. It is complemented by a five-layer Pixel Detector with the coverage extended to $|\eta|<4$. To cope with the radiation damage, the innermost two layers of the Pixel Detector will be replaceable so as to be replaced after half of the integrated luminosity is accumulated. The total surface area of the pixel 
detector is $13 \mathrm{~m}^{2}$ with approximately $5 \times 10^{9}$ channels, and the area of the Strip Detector is $165 \mathrm{~m}^{2}$ with approximately $\sim 6 \times 10^{7}$ channels. The benchmark layout of ITk [6] allows a track quality cut of at least 7-9 silicon hits per track in the offline analysis level, depending on the pseudo-rapidity of the tracks. Figure 1 (a) shows that the efficiency for $10 \mathrm{GeV}$ muon tracks is almost $100 \%$ for the entire area and $90-98 \%$ of efficiency for electrons and pions with the quality cut. Interactions with detector materials limit the efficiency of the electrons and pions compared with muons, due to Bremsstrahlung radiations of electrons and inelastic hadronic interactions of pions, respectively. Figure 1 (b) shows the fake rate evaluated for the quality cut with simulated data, where the fake rate is defined as the fraction of reconstructed tracks which are not associated with truth particles. The ITk results are compared to the Run 2 detector performance. Although a much higher number of pileup collisions is assumed for ITk performance studies, ITk shows better performance of fake rates. It is owing to the enlarged lever arm and higher granularity on the Pixel and Strip Detectors.

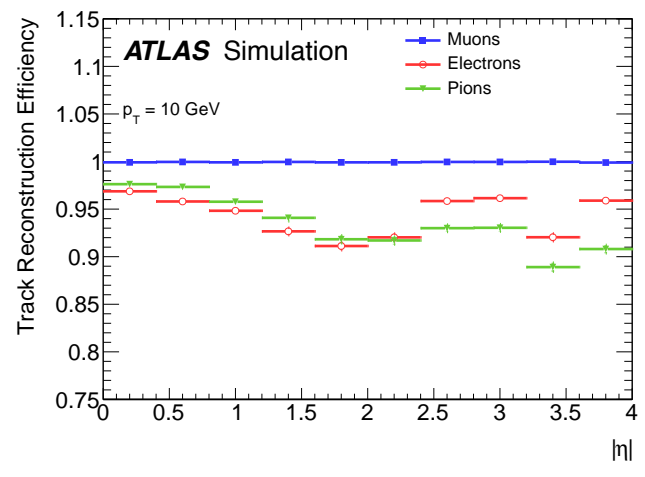

(a)

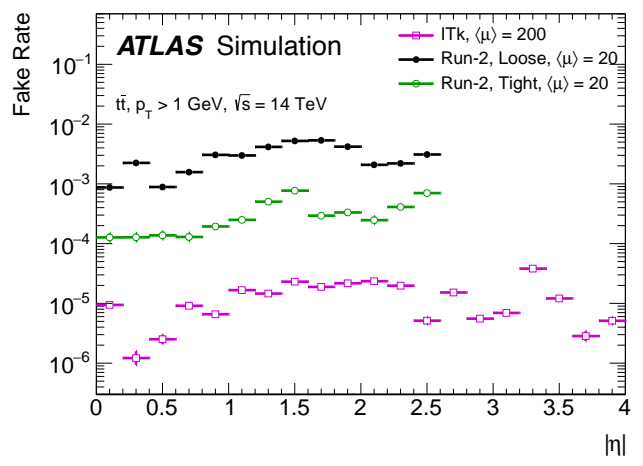

(b)

Figure 1: (a) Track reconstruction efficiency for single muons, pions, and electrons with a fixed transverse momentum of $10 \mathrm{GeV}$. (b) Fake rate based on the truth particle matching with ITk system $(|\eta|<4.0)$ as well as the Run 2 detector system $(|\eta|<2.5)$. The Run 2 performance is shown with respect to the standard two track selection criteria of "Loose" and "Tight" [6].

Vertex reconstruction capability is another important requirement on the ITk performance. Figure 2 (a) and (b) show the resolution of the primary vertex reconstruction in $z$ position, and the primary vertex reconstruction efficiency for $t \bar{t}$ and $H \rightarrow Z Z^{*} \rightarrow v v v v$ with Vector Boson Fusion (VBF) production process, respectively. In both figures, the performance is shown as a function of local pileup density. Comparing the tracking performance of ITk to the Run 2 detector performance, it is shown that ITk will have a robust performance of the vertex reconstruction.

In order to distinguish collisions of interest from other pileup vertices, the Phase-2 ATLAS system will exploit the time spread of the collisions in each bunch crossing. Given that a pair of two finite size bunches cross with a light velocity, as shown in Figure 3 (a), the time spread of $\sim 175$ ps is expected with the nominal operation scheme of the HL-LHC. The precise timing information for individual charged tracks can give additional separation powers of the collision points as long as the timing resolution is good enough with respect to the expected time spread of collisions per bunch crossing. For this purpose, a new timing detector for charged tracks, High-Granularity TimingDetector (HGTD), will be installed in the Phase-2 system [8]. The HGTD will cover the forward 


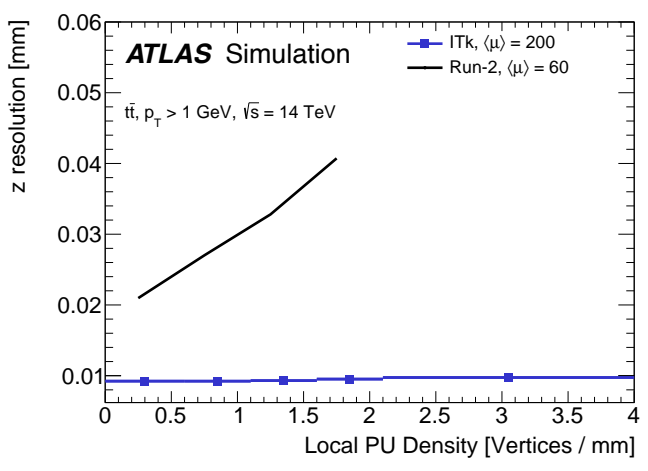

(a)

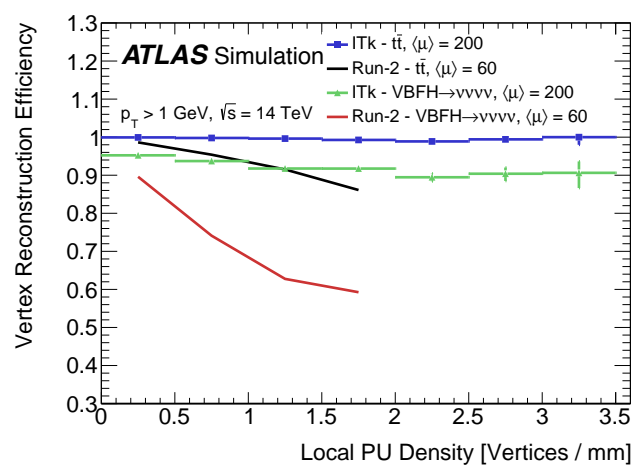

(b)

Figure 2: (a) The resolution in $z$ measurement of vertex reconstruction with $t \bar{t}$ process. (b) The primary vertex reconstruction efficiency for $t \bar{t}$ and $\mathrm{VBF} H \rightarrow Z Z^{*} \rightarrow v v v v$ processes. Both the resolution and efficiency are shown as a function of local pileup density [6].

region $(2.4<|\eta|<4.0)$ of ATLAS detector with double-sided planar silicon pixel sensors. The size of the pixel is $1.3 \times 1.3 \mathrm{~mm}^{2}$ and the thickness is $50 \mu \mathrm{m}$. The timing resolution of the pixel sensors due to intrinsic Landau fluctuations is expected to be $\sim 25 \mathrm{ps}$. Taking additional timing jitters due to electronics ( $\sim 25 \mathrm{ps})$ and clock distributions ( $\sim 10 \mathrm{ps})$ into account, the expected timing resolution for minimum-ionizing particles is $\sim 30-50 \mathrm{ps}$. In order to optimize the detector design, performance studies have been done in terms of object reconstruction, luminosity measurement, and application in trigger algorithms. As an example, Figure 3 (b) shows the performance of an algorithm to suppress pileup jets with HGTD information. The rejection factor of jets from pileup collisions is shown as a function of the efficiency for jets from the collisions of interest, with respect to jets reconstructed in a phase-space of $2.4<|\eta|<4.0$ and $30 \mathrm{GeV}<p_{\mathrm{T}}<50 \mathrm{GeV}$. It demonstrates that an algorithm based on the timing information given by the HGTD can provide additional rejection for the jets originated from the pileup collisions.

\subsection{New TDAQ system}

The TDAQ system will be upgraded for the Phase-2 system through LS2 and LS3 to sustain the stability and of the data-taking and flexibility in the triggering in the high luminosity environment [12]. Figure 4 (a) is the schematics of the TDAQ system, equipped with Level-0 hardwarebased trigger (L0), Event Filter software-based trigger (EF), and the permanent storage. The baseline architecture of the Phase-2 TDAQ system features a maximum L0 readout rate of $1 \mathrm{MHz}$ and $10 \mu$ s fixed latency of the L0 trigger algorithms. The Event Filter software-based trigger follows the L0 trigger selection to achieve further rejection. The final recording rate to the permanent storage will be up to $10 \mathrm{kHz}$. The system is designed to sustain the capability of efficient selections in the triggering stage for physics within or beyond the Standard Model in the HL-LHC environment with enough flexibility. This section will present the Phase-2 TDAQ system, focusing on the L0 muon trigger upgrade and the new hardware-based track trigger system.

The Phase-2 upgrade of the TDAQ system takes advantage of modern Field Programmable Gate Array (FPGA) devices and cost-effective high-bandwidth optical links to cope with the re- 


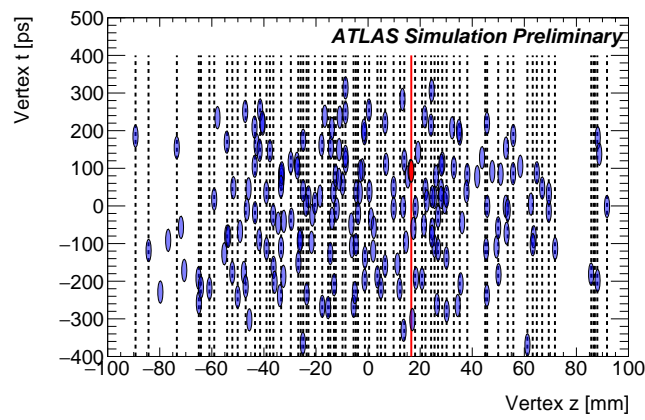

(a)

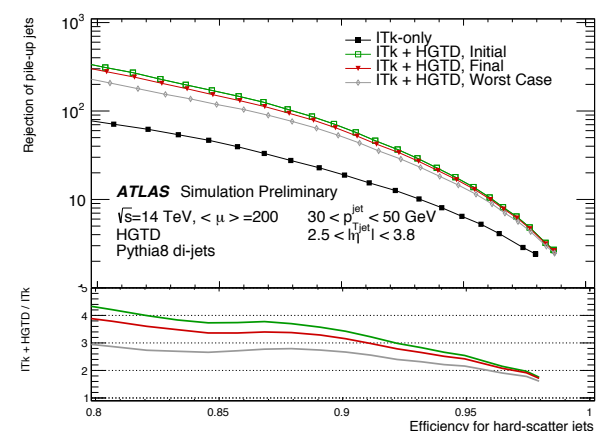

(b)

Figure 3: (a) The primary vertices in a bunch crossing in the $z-t$ plane with a vertex of interest (red ellipse) and about 200 pileup interactions (blue ellipses) superimposed. (b) Rejection of jets originated from pileup collisions as a function of efficiency for jets originated from the vertex of interest for ITk-only and ITk+HGTD scenarios [8].

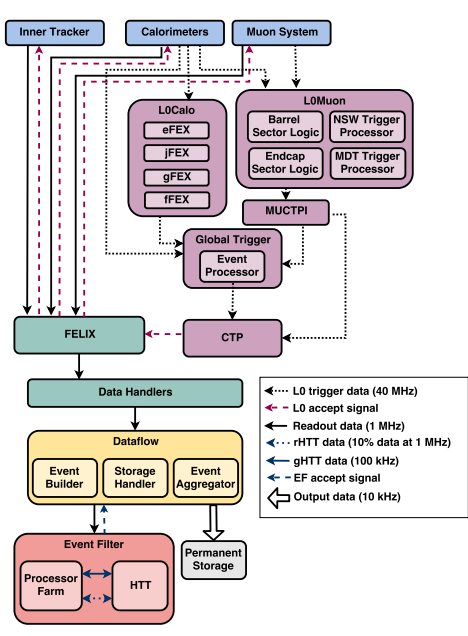

(a)

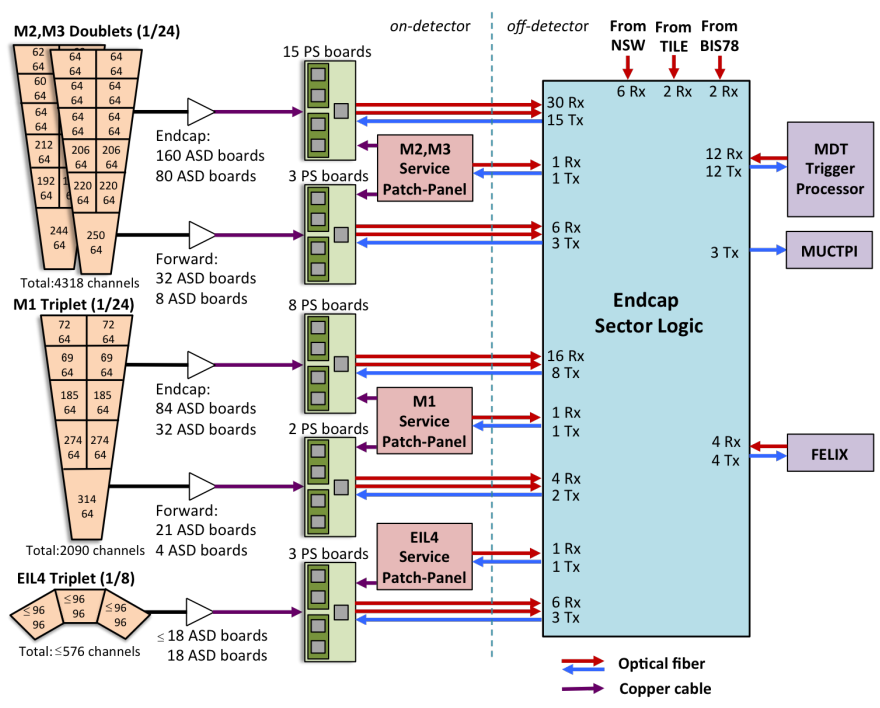

(b)

Figure 4: (a) Design of the upgraded TDAQ system architecture [12]. (b) Diagram of the upgraded L0 endcap muon trigger system. Fully exploiting the increased bandwidth available on the on-detector electronics, all the hits can be sent to the back-end electronics without pre-selection [9].

quirements on the latency and trigger rate. As an example of such upgrade programs, the L0 endcap muon trigger system, which covers $|\eta|>1.05$, will upgrade the electronics system as shown in Figure 4 (b) with modern high-bandwidth serial links and FPGAs on the front-end electronics (PS Board), and large resources of logic gates and RAM blocks in the FPGAs on the back-end electronics (Sector Logic). With the Phase-2 L0 endcap muon trigger system, it will be possible to send binary hit information for all the individual channels at every bunch crossing to the back-end electronics. Figure 5 (a) shows the block diagram of the new front-end PS Board equipped with 
a modern FPGA and high-bandwidth optical links. Owing to the capability of sending all binary hits to the back-end Sector Logic board, a sophisticated pattern matching algorithm to find a muon candidate can be implemented in the back-end electronics. Figure 5 (b) demonstrates the improvement of the trigger efficiency turn-on curve with the pattern matching algorithm, compared with the performance seen in the Run 1 system.

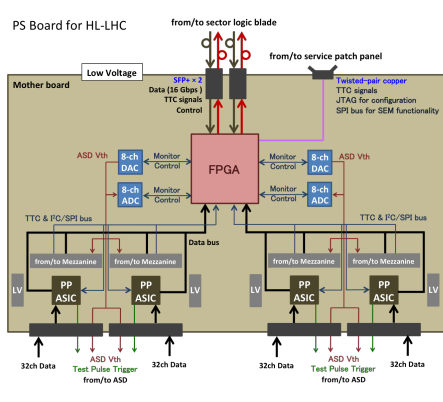

(a)

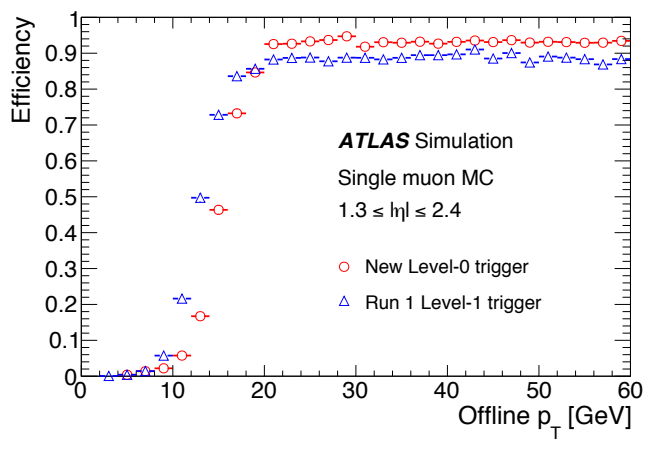

(b)

Figure 5: (a) Block diagram of the new front-end electronics of the muon trigger system [9]. (b) Expected efficiency for the upgraded muon trigger in the $1.3<|\eta|<2.4$ for a $p_{\mathrm{T}}$ threshold of $20 \mathrm{GeV}$. The expected performance with the HL-LHC scheme (red points) as well as with the Run 1 system (blue points) is shown [12].

The Phase-2 TDAQ system will sustain the capability to implement new algorithms, taking advantage of the extended latency of $10 \mu \mathrm{s}$. Monitored Drift-Tube (MDT) detector is a precision muon detector. Although, due to the long maximum drift-time of $\sim 700 \mathrm{~ns}$, the MDT has been only used for precision tracking since the LHC started, the longer latency of the Phase-2 L0 system will make it feasible for the MDT to be involved in the L0 muon trigger algorithm to improve the momentum resolution of the L0 muon trigger, which is called L0 MDT system. The algorithm of the L0 MDT processors consists of two steps; (1) straight "track segment" reconstruction at each MDT detector layer of the three layers, and (2) momentum estimation by the combination of the segments of three layers. Further control of trigger rate with a sharper turn-on curve will be gained as shown in Figure 6 (a) with the use of the precise muon tracking information given by the new L0 MDT processors. The straight track segment reconstruction is the major technological challenge of the new system, and currently, the Legendre Transformation on FPGAs and the Associative Memory ASICs are considered as candidate technologies. Figure 6 (b) shows the segment reconstruction efficiency at an MDT layer with the Associative Memory approach.

A new system of Hardware-based Tracking for the Trigger (HTT) will be introduced to provide tracks to software-based Event Filter. The fast-tracking relies on the track-finding using the Associative Memory ASICs followed by a track-fitting algorithm implemented in FPGAs. The HTT includes two complementary functionalities for a regional (regional HTT; rHTT) and fullscan (global HTT; gHTT) track reconstruction capabilities. The rHTT will perform the tracking at the full $\mathrm{L} 0$ rate of $1 \mathrm{MHz}$ in regions of interest, which corresponds to $10 \%$ of ITk data in these events. The rHTT will find tracks with $p_{\mathrm{T}}>2 \mathrm{GeV}$ in the limited regions of interest pointed by the L0 trigger algorithms. It will improve the identification and the resolution of the L0 trigger objects, 


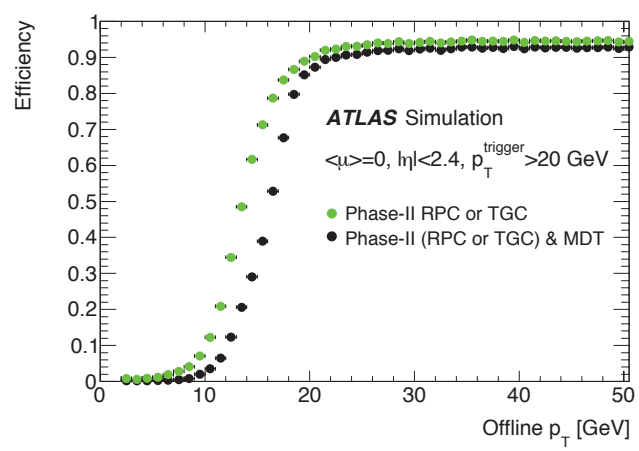

(a)

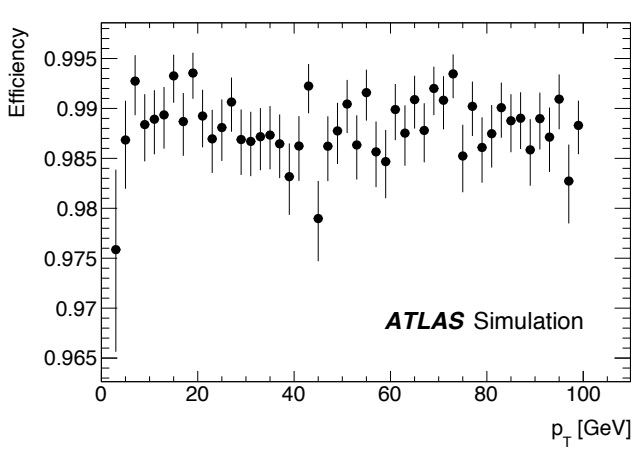

(b)

Figure 6: (a) Trigger efficiency for muons with $|\eta|<2.4$ as a function of the transverse momentum $\left(p_{T}\right)$ measured by the offline reconstruction. It demonstrates the improvement expected by the introduction of new L0 MDT system in the L0 muon trigger. (b) Track segment reconstruction efficiency estimated by the simulation of the Associative Memory based system [12].

such as single high- $p_{T}$ leptons, and allow early rejection of the backgrounds at the beginning of the Event Filter algorithm to reduce the rate to around $400 \mathrm{kHz}$. Figure 7 (a) shows the performance of the early rejection for the electrons, based on the L0 electromagnetic calorimeter objects. The rejection that can be gained by a requirement of an associated track with the $p_{\mathrm{T}}>20 \mathrm{GeV}$ is shown as a function of the efficiency. The rejection with a factor of $\sim 5$ is expected while $95-97 \%$ efficiency for an online electron threshold of $18 \mathrm{GeV}$. The gHTT will perform full-scan tracking at a nominal rate of $100 \mathrm{kHz}$ so as to reconstruct the event-level information for the events. The gHTT will reconstruct tracks with $p_{\mathrm{T}}>1 \mathrm{GeV}$ in the full detector regions. The online missing transverse energy $\left(E_{\mathrm{T}}^{\text {miss }}\right)$ reconstruction is to be improved exploiting both rHTT and gHTT. While the rHTT output is used in order to require the jets in the $E_{\mathrm{T}}^{\text {miss }}$ calculation to be originated from the primary vertex of interest, the gHTT output is used to calculate a sum of all tracks in the full detector coverage with a requirement to share the common primary vertex. Figure 7 (b) demonstrates the expected improvement in the online $E_{\mathrm{T}}^{\text {miss }}$ reconstruction with the assistance of rHTT and gHTT. Background rate is shown as a function of trigger threshold in terms of offline $E_{\mathrm{T}}^{\text {miss }}$ at which the trigger efficiency is as high as $95 \%$. Various scenarios of online tracking acceptance are tested. In a wide range of $E_{\mathrm{T}}^{\text {miss }}$, additional rejection with a factor of 10-100 is expected with the assistance of the HTT.

\section{Summary}

The high instantaneous luminosity of the HL-LHC will be realized by a full upgrade of the components of the LHC accelerator complex such as the injector chain, the collimators, and the elements in the insertion regions for the ATLAS and CMS experiments. A luminosity levelling technique will be introduced in order to control energy deposit at the magnets in the interaction regions and for a smooth operation of the detector systems. The LHC-ATLAS experiment will introduce new detectors, electronics, and trigger components to sustain the high performance of the reconstruction of the proton-proton collision events. The new charged particle tracking sys- 


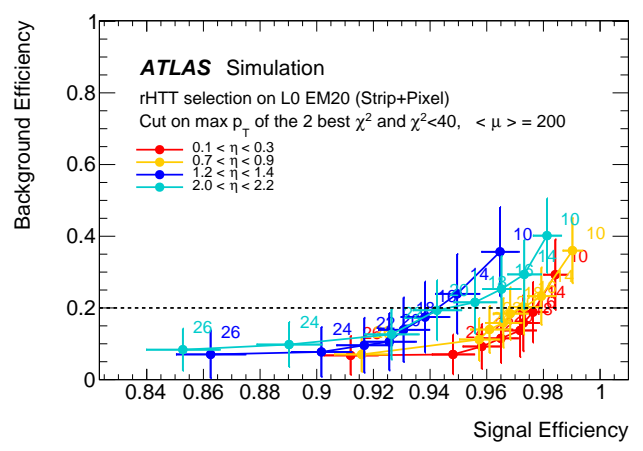

(a)

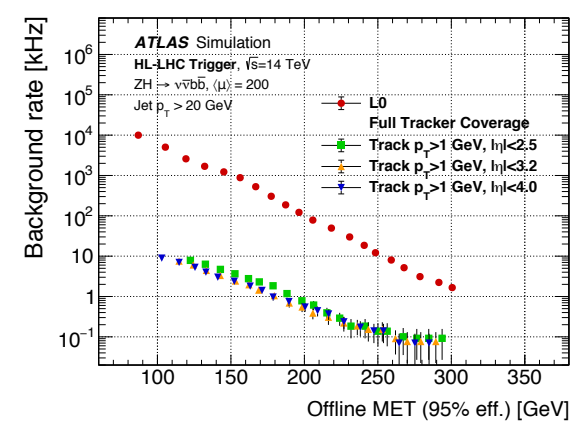

(b)

Figure 7: (a) Rejection of the backgrounds with a track requirement for electrons as a function of efficiency. (b) Background rate of the $E_{\mathrm{T}}^{\text {miss }}$ trigger as a function of the threshold with HTT for various scenarios of the HTT tracking acceptance in $\eta$, compared with the performance of the L0 based $E_{\mathrm{T}}^{\text {miss }}$ calculation (red) [12].

tem (ITk) and High-Granularity Timing-Detector (HGTD) are the new detectors to be installed to cope with 200 pileup collisions per bunch crossing. The TDAQ system upgrade will allow the experiment to apply sophisticated algorithms in the online triggering stage and readout the expected larger data volume. New algorithms will be introduced in the hardware trigger system, such as pattern matching based L0 muon algorithm and L0 MDT system. The HTT, a new hardware-based tracking system, will be used to support the $1 \mathrm{MHz}$ L0 trigger rate and for the flexibility of the trigger algorithm in the Event Filter.

\section{Acknowledgement}

This work was partially supported by JSPS KAKENHI Grant Number 17H02880.

\section{References}

[1] ATLAS Collaboration, 2008 JINST 3 S08003.

[2] CMS Collaboration, 2008 JINST 3 S08004.

[3] L. Evans and P. Bryant, 2008 JINST 3 S08001.

[4] Apollinari, G. et al., CERN Yellow Rep.Monogr. 4 (2017) 1-516 CERN-2017-007-M.

[5] The HL-LHC Project, https://project-hl-lhc-industry •web.cern.ch/

[6] ATLAS Collaboration, CERN-LHCC-2017-021; ATLAS-TDR-030.

[7] ATLAS Collaboration, CERN-LHCC-2017-005 ; ATLAS-TDR-025.

[8] ATLAS Collaboration, CERN-LHCC-2018-023 ; LHCC-P-012.

[9] ATLAS Collaboration, CERN-LHCC-2017-017 ; ATLAS-TDR-026.

[10] ATLAS Collaboration, CERN-LHCC-2017-018; ATLAS-TDR-027.

[11] ATLAS Collaboration, CERN-LHCC-2017-019; ATLAS-TDR-028.

[12] ATLAS Collaboration, CERN-LHCC-2017-020 ; ATLAS-TDR-029. 